\title{
SURFACE AND THIN FILM MAGNETIZATION OF ISOTROPIC AND ANISOTROPIC HEISENBERG FERROMAGNET AT LOW TEMPERATURES
}

\author{
P. MODRAK* \\ Institute of Physical Chemistry, Polish Academy of Sciences \\ Kasprzaka 44/52, 01-224 Warsaw, Poland
}

(Received January 26, 1998)

\begin{abstract}
The independent random walk method (which is equivalent to the noninteracting spin wave method) with no further approximations was used to calculate the low tęmperature expansion of: (i) the local mean spin values of an isotropic Heisenberg ferromagnet for semi-infinite crystal and (ii) the spontaneous magnetization of an anisotropic Heisenberg ferromagnet for monolayer and double layer. The full low temperature expansion starting with $T^{3 / 2}$ term was obtained for the semi-infinite Heisenberg ferromagnet and it was shown that the spontaneous magnetization for thin films of anisotropic Heisenberg ferromagnets can exhibit a quasi-linear behaviour in certain temperature region related to the magnitude of the anisotropy.
\end{abstract}

PACS numbers: 75.70.Ak, 75.30.Pd

\section{Introduction}

Magnetic thin films and multilayers exhibit various interesting properties different from bulk ferromagnets [1]. The experimental data on the behaviour of the magnetization of some overlayers in the low temperature region show, for example, the appearance of a linear term in the low temperature expansion of the surface magnetization of the ferromagnets [2]. The theory of the influence of the surface on the magnetization of the isotropic Heisenberg ferromagnet [3] predicts only the change of the $T^{3 / 2}$ term (where $T$ is the absolute temperature) with the prefactor of the term twice as large as the bulk one. The analysis of the spin-wave spectra for the Heisenberg ferromagnet with exchange softened at the surface or in overlayers leads to the conclusion that the magnetization of the systems obeys with a good approximation the $T^{3 / 2}$ law $[4,5]$ while the $T^{3 / 2}$ prefactor can vary [5] in a wide region. The enhancement of the exchange in the

*e-mail: modrak@ichf.edu.pl 
surface layer or the surface spin also affects the surface magnetization [5] and its temperature expansion for $J_{\|}=3 J$ (where $J$ and $J_{\|}$are the bulk exchange and the exchange in the surface layer, respectively) can be considered as a combination of linear and $T^{3 / 2}$ dependence.

The magnetization of ultrathin films has been intensively studied both experimentally and theoretically (see Ref. [6] and [7], respectively, and references therein). The ferromagnetism absent in two-dimensional isotropic Heisenberg model [8] can be restored by an arbitrary small anisotropy [9] and a magnetic monolayer can undergo various phase transitions [7].

The present paper is confined to thermodynamic properties of the Heisenberg ferromagnets in low temperature region. The investigation of the surface and thin film magnetization is based on the random walk technique [10]. The method, due to Kramers [11], consists in the direct calculation of the partition function and does not make use of Bloch's spin waves. The partition function is calculated in the basis of the localized states and such a basis is certainly suitable for the crystal with the surface for which the translational symmetry is broken.

The present paper has two objectives. First, it will be demonstrated that the low temperature expansion of the spin mean deviation for the surface and undersurface layers of the semi-infinite isotropic Heisenberg ferromagnet can be derived in the exact manner within the scope of the noninteracting spin-wave approximation. The coefficient of the first term of the expansion agrees with the one previously obtained [3]. Then it will be shown that even a small exchange anisotropy can restore ferromagnetic order (in agreement with Ref. [9]) both in mono- and double layers. It will be also shown that the spontaneous magnetization for the thin film anisotropic Heisenberg ferromagnet exhibits the quasi-linear behaviour in a certain temperature region depending on the magnitude of the anisotropy. The exchange anisotropy can thus provide an alternative way of explanation of the quasi-linear behaviour of thin film magnetization in the low temperature region.

All calculations have been performed for the simple cubic (sc) lattice. The form of the expansion obtained (except for numerical values of coefficients) should not depend on the symmetry of the lattice and the calculations are simplified by the fact that the random walk can be decomposed into one-dimensional walks for the sc lattice.

\section{Semi-infinite Heisenberg ferromagnet}

We assume that we have a cubic lattice with the (001) surface plane which consists of $N$ lattice sites. The positions of lattice sites belonging to the surface plane are assumed to be $j=\left(n_{x}, n_{y}, 1\right)\left(n_{x(y)}=0, \pm 1, \ldots\right.$ and the unit of the distance is the lattice constant). A homogeneous magnetic field directed along the positive $z$ axis is supposed to be present. First, we consider the system for which each atom carries a spin $S=1 / 2$, the generalization for an arbitrary $S$ is straightforward. Neglecting all except nearest-neighbour interactions, the Heisenberg ferromagnet can be written in the following form:

$$
\widehat{H}=J\left(\epsilon+\epsilon_{H}\right)
$$




$$
\begin{aligned}
& \epsilon=-\sum_{\boldsymbol{j}_{1} \boldsymbol{j}_{2}}\left(1+\sigma_{\boldsymbol{j}_{1}} \sigma_{\boldsymbol{j}_{2}}\right), \\
& \epsilon_{H}=-a \sum_{\boldsymbol{j}} \sigma_{z \boldsymbol{j}}, \quad a=\mu H / J,
\end{aligned}
$$

where $J$ is the exchange interaction constant $(J>0), \sigma_{j}$ is the Pauli spin-vector operator of the $j$-th atom, $\mu$ and $H$ are the magnitudes of the magnetic moment and the magnetic field, respectively, and the sum in Eq. (2.2) extends over all $N f$ pairs of adjacent lattice sites and the sum in Eq. (2.3) extends over all $N$ atoms. The partition function $Z$ can be written in the form $[10,12]$

$$
Z=\operatorname{Tr} \exp \left[-\beta\left(\epsilon+\epsilon_{H}\right)\right]=\sum_{n=0}^{\infty}\left(\beta^{n} / n !\right) \sum_{l=0}^{n}\left(\begin{array}{l}
n \\
l
\end{array}\right) \operatorname{Tr}\left[(-\epsilon)^{l}\left(-\epsilon_{H}\right)^{n-l}\right],
$$

where $\beta=J / k T$ and $k$ is the Boltzmann constant. The local mean spin value at the position $j$ we want to calculate is given by the formula

$$
\left\langle S_{z, \boldsymbol{j}}\right\rangle=\frac{1}{2}\left\{Z^{-1} \operatorname{Tr} \exp \left[-\beta\left(\epsilon+\epsilon_{H}\right)\right] \sigma_{z j}\right\}_{a \Rightarrow 0} .
$$

The traces appearing in Eq. (2.5) will be calculated in the representation in which $\epsilon_{H}$ is diagonal. The function in this representation will be fully defined by stating at which lattice sites there are spins oriented in the direction opposite to the direction of the magnetic field (such spins will be called minus-spins) and will be denoted by $|m, u\rangle$, where $m$ is the number of minus-spins and $u$ describes their configuration. We can write

$$
(-\epsilon)^{l}\left(-\epsilon_{H}\right)^{n-l}\left|m, u_{0}\right\rangle=a(N-2 m)^{n-l}(-\epsilon)^{l}\left|m, u_{0}\right\rangle .
$$

and

$$
-\epsilon\left|m, u_{0}\right\rangle=A_{m, u_{0}}|m, u\rangle+\sum_{u_{1}}\left|m, u_{0}, u_{1}\right\rangle .
$$

The result is a simple consequence of the fact that $(1 / 2)\left(1+\sigma_{j_{1}} \sigma_{j_{1}}\right)$ is equivalent to the permutation operator exchanging the spin states of the atoms $j_{1}$ and $j_{2}$. The state $\left|m, u_{0}, u_{1}\right\rangle$ is the eigenstate obtained from $\left|m, u_{0}\right\rangle$ by the displacement of one of $m$ minus-spins from one lattice point to the adjacent lattice point. $u_{0}$ is kept in the symbol $\left|m, u_{0}, u_{1}\right\rangle$ to show the way in which the eigenstate arose. $A_{m, u}$ is simply the number of pairs of adjacent lattice sites which are either both occupied or both unoccupied by minus-spins. The approximation introduced in the calculation for an infinite crystal [10] consists in the assumption that $A$ can be calculated as if no two minus-spins ever occupied adjacent lattice points. In this approximation, for infinite ferromagnet,

$$
A_{m, u}=N f-2 f m \text {. }
$$

The approximation regarding the mutual position of minus-spins, well justified for the low temperature region, is also introduced in the present calculation. The effect of the surface is, however, taken into account without any approximations.

The presence of the surface can change the value of $A_{m, u}$. This value defining the number of pairs of adjacent lattice sites which are both unoccupied by minus-spins depends now on the number of minus-spins in the surface layer. In 
order to account for this effect, let us introduce first, similarly as in Ref. [12], the operator $\widehat{n}_{\boldsymbol{j}}$ defined by its effect on the state $|m, u\rangle$ in the following way:

$$
\widehat{n}_{j}|m, u\rangle \doteq n|m, u\rangle,
$$

where $n$ is the number of minus-spins occupying the $j$-th lattice site. It is also convenient to introduce the operator $\widehat{n}_{j, u_{k}}$ defined by the equation

$$
\begin{aligned}
& \widehat{n}_{j, u_{k}}\left|m, u_{0}, \ldots u_{k}, \ldots u_{f}\right\rangle \\
& \quad=\left\langle m, u_{0}, \ldots u_{k}\left|\widehat{n}_{\boldsymbol{j}}\right| m, u_{0}, \ldots u_{k}\right\rangle\left|m, u_{0}, \ldots u_{k}, \ldots u_{f}\right\rangle
\end{aligned}
$$

and the operator $\widehat{n}_{\boldsymbol{j}, u_{k}}^{l}$ defined in the following way:

$$
\widehat{n}_{j, u_{k}}^{l}\left|m, u_{0}, \ldots u_{k}, \ldots u_{f}\right\rangle=\delta\left|m, u_{0}, \ldots u_{k}, \ldots u_{f}\right\rangle
$$

where $\delta$ equals 1 when the $l$-th minus-spin occupies in the configuration $u_{k}$ the $j$ lattice site and 0 otherwise. $\widehat{n}_{\boldsymbol{j}, u_{k}}$ and $\widehat{n}_{\boldsymbol{j}, u_{k}}^{l}$ are related by the equation

$$
\widehat{n}_{j, u_{k}}\left|m, \iota_{0}, \ldots u_{k}, \ldots u_{f}\right\rangle=\sum_{l=1}^{m} \widehat{n}_{j, u_{k}}^{l}\left|m, u_{0}, \ldots u_{k}, \ldots u_{f}\right\rangle .
$$

$A_{m, u_{k}}$ can be now replaced with the operator $\widehat{A}_{m, u_{k}}$ and the latter can be expressed in terms of the operator $\widehat{n}_{j, u_{k}}^{l}$

$$
\widehat{A}_{m, u_{k}}=A_{m}+f_{\mathrm{d}} \widehat{n}_{u_{k}},
$$

where

$$
\begin{aligned}
& A_{m}=N f-\frac{1}{2} K f_{\mathrm{d}}-2 f m, \\
& \widehat{n}_{u_{k}}=\sum_{\boldsymbol{j} \subset S} \widehat{n}_{\boldsymbol{j}, u_{k}},
\end{aligned}
$$

$f_{\mathrm{d}}$ is the number of dangling bonds at the surface, $K$ is the number of atoms in the surface layer and the summation in Eq. (2.15) is over all $j$ belonging to the surface plane. $\sigma_{z \boldsymbol{j}}$ can be also related to the operator $\hat{n}_{\boldsymbol{j}, u_{k}}^{l}$

$$
\sigma_{z j}=1-2 \widehat{n}_{\boldsymbol{j}, u_{0}} .
$$

The calculation of the partition function is now reduced formally to the same problem as was considered in Ref. [12]. The only difference is that the operators $\widehat{n}_{\boldsymbol{j}}$ are used now to find the number of minus-spins in the surface layer instead of the number of minus-spins in the vicinity of nonmagnetic impurities considered in Ref. [12]. We can therefore use the result of Ref. [12] to write

$$
Z=\sum_{m=0}^{N} \exp \left[\beta\left(h_{m}+A_{m}\right)\right] \sum_{t=0}^{\infty} \sum_{l=0}^{\infty} \frac{\beta^{l+t}}{(l+t) !} \sum_{u_{0}} s_{l}^{t}\left(m, u_{0}\right),
$$

where

$$
\begin{aligned}
& h_{m}=a(N-2 m), \\
& s_{l}^{t}\left(m, u_{0}\right)=\sum_{u_{1} \ldots u_{l}} f_{d}^{t}\left\langle m, u_{0}\left|\sum_{k_{t} \geq \ldots \geq k_{1} \geq 0}^{l} \widehat{n}_{u_{k_{1}}} \ldots \widehat{n}_{u_{k_{t}}}\right| m, u_{0}, \ldots, u_{l}\right\rangle .
\end{aligned}
$$


The calculation of the partition function for infinite crystals [10, 12] was reduced at this stage to solving a random walk problem. The simplification made consisted in the assumption that $m$ minus-spins perform their random walk independently and two or more minus-spins may occupy occasionally the same lattice site. The present calculations are based on the same simplifying assumption. The calculation for infinite crystals [10] based on the independent random walk approximation and the approximate calculation of $A_{m}$ give the low temperature expansion of the spontaneous magnetization with the accuracy up to (and including) $T^{7 / 2}$ term.

The calculation of $s_{l}^{t}\left(m, u_{0}\right)$ differs from the calculation for infinite crystals containing nonmagnetic impurities [12] only in one point. No trajectory of a minus-spin for the infinite crystal can pass through the surface plane instead of the lattice sites occupied by nonmagnetic impurities [12]. The probability that a minus-spin initially at $j_{1}$ arrives after $l$ steps at $j_{2}$ not passing through the surface plane is given by $[13,14]$

$$
T_{l}\left(j_{1}, j_{2}\right)=P_{l}\left(j_{1}, j_{2}\right)-P_{l}\left(j_{1}, j_{2}{ }^{\prime}\right)
$$

where $P_{l}$ is the probability that a minus-spin initially at $j_{1}$ arrives after $l$ steps at $j_{2}$ passing or not passing through the surface and $j_{2}{ }^{\prime}$ is the symmetrical site of $j_{2}$ with regard to the first fictitious lattice plane parallel to the surface (Eq. (2.20) is valid provided that the surface plane is a symmetry plane of the crystal).

The probability $P_{l}\left(j_{1}, j_{2}\right)$ is easy to calculate [10]

$$
P_{l}\left(j_{1}, j_{2}\right)=\Omega^{-1} \int R_{\rho}^{l} \exp \left[\mathrm{i} \rho\left(j_{2}-j_{1}\right)\right] \mathrm{d} \rho,
$$

where

$$
R_{\rho}=\frac{1}{2 f} \sum_{k} \exp \left(\mathrm{i} \rho r_{k}\right)
$$

the sum in (2.22) is over all adjacent lattice sites with regard to any given lattice site, $\rho$ is a vector of the reciprocal lattice, the integration in (2.21) is over the unit cell of the reciprocal lattice and $\Omega$ is its volume.

It can be easily verified that for the sc lattice with the (001) surface plane

$$
\sum_{j_{2}} T_{l_{1}}\left(j_{1}, j_{2}\right) T_{l_{2}}\left(j_{2}, j_{3}\right)=T_{l_{1}+l_{2}}\left(j_{1}, j_{3}\right) \text {. }
$$

The above relation allows us to use all results obtained in Ref. [12]. The relation (2.16) together with (2.12) shows that the presence $\sigma$ under the trace fixes only the positions of minus-spins in the initial configuration and beside that all calculations can be performed in the same way as in the case of the partition function. Using the results of Ref. [12] without any further approximation we can obtain after some rearrangements (see Appendix)

$$
\left\langle S_{z, l}\right\rangle=S-\sum_{q=1}^{\infty} \exp (-\gamma) \sum_{t=0}^{\infty}(2 \pi)^{-t-3} \int \mathrm{d} \rho_{x} \mathrm{~d} \rho_{y} \mathrm{~d} \rho_{z_{1}} \ldots \mathrm{d} \rho_{z_{t+1}} \mathcal{P}_{l} C_{t}
$$

where

$$
C_{t}=\exp \left(\gamma R_{p}\right) \alpha^{t} \sum_{i_{1} \ldots i_{t+1}=0}^{\infty} \frac{\left(\gamma R_{z_{1}}\right)^{i_{1}} \ldots\left(\gamma R_{z_{t+1}}\right)^{i_{t+1}}}{\left(i_{1}+\ldots+i_{t+1}+t\right) !}
$$




$$
\begin{aligned}
\mathcal{P}_{l}= & \exp \left[\mathrm{i} \rho_{z_{1}}(l-1)\right] \\
& \times \prod_{k=1}^{t}\left[1-\exp \left(2 \mathrm{i} \rho_{z_{k}}\right)\right] \exp \left[-\mathrm{i} \rho_{z_{t+1}}(l-1)\right]\left[1-\exp \left(2 l \mathrm{i} \rho_{z_{t+1}}\right)\right], \\
\alpha= & 2 S q \beta, \\
\gamma= & 2 f \alpha, \\
R_{p}= & f^{-1}\left(\cos \rho_{x}+\cos \rho_{y}\right), \\
R_{z}= & f^{-1} \cos \rho_{z},
\end{aligned}
$$

$l=1$ denotes the surface plane and $l=2,3 \ldots$ the successive undersurface planes. Making use of the following representation of the reciprocal of $n$ !

$$
(n !)^{-1}=(2 \pi)^{-1} \exp (\eta) \int_{-\infty}^{\infty} \mathrm{d} x(\eta-\mathrm{i} x)^{-n-1} \exp (-\mathrm{i} x)
$$

we finally get for the surface layer (see Appendix)

$$
\left\langle S_{z, 1}\right\rangle=S-\sum_{q=1}^{\infty} \exp [-2 f(2 \alpha)] I_{0}(2 \alpha)^{2} B(\alpha)
$$

where

$$
B(\alpha)=\exp (2 \alpha) \int_{-\infty}^{\infty} \mathrm{d} x \exp (-2 \pi \mathrm{i} \alpha x)\left(\frac{\sqrt{x+2 \mathrm{i}}}{\sqrt{x}}-1\right)
$$

and $I_{0}$ is the Bessel function.

$\alpha$ being proportional to $T^{-1}$ is large in the low temperature region. The asymptotic expansion of the Bessel function for a large variable is known and the asymptotic expansion of the integral $B$ can be obtained following the method described in Ref. [15]. The low temperature expansion of $\left\langle S_{z, 1}\right\rangle$ can be finally written in the following form:

$$
\left\langle S_{z, 1}\right\rangle=S-b T^{3 / 2}-c T^{5 / 2}-\ldots
$$

where

$$
\begin{aligned}
& b=\frac{\zeta(3 / 2)}{4 \pi^{3 / 2}(2 S J / k)^{3 / 2}}, \\
& c=\frac{\zeta(5 / 2)}{128 \pi^{3 / 2}(2 S J / k)^{5 / 2}}\left(4-2^{3 / 2}\right)
\end{aligned}
$$

and $\zeta$ is the Riemann zeta function. The expansion (2.34) should be exact up to the $T^{-7 / 2}$ term. The coefficient $b$ and $c$ can be compared with the coefficient $b_{\infty}$ and $c_{\infty}$ for the infinite crystal

$$
b=2 b_{\infty}
$$

(in agreement with Ref. [3]) and

$$
c=\frac{\left(4-2^{3 / 2}\right)}{3} c_{\infty} .
$$


The low temperature expansion of the mean spin deviations thus obtained is exact within the scope of the noninteracting spin-wave approximation. The calculation shows that the presence of the surface affects only numerical values of coefficients of the expansion and does not explain the appearance of a linear term. However, the presence of the surface can affect the coupling between spins introducing an anisotropy in the exchange interaction. The spontaneous magnetization of thin films of anisotropic Heisenberg ferromagnets will be considered in the following section.

\section{Thin films of anisotropic Heisenberg ferromagnet}

Let us write the Hamiltonian of the anisotropic Heisenberg ferromagnet for $S=1 / 2$ in the following form:

$$
\widehat{H}=\widehat{H}_{0}+\widehat{H}^{\prime},
$$

where $\widehat{H}_{0}$ is given by Eq. (2.1)

$$
\widehat{H}^{\prime}=-J \Delta J \frac{1}{2} \sum_{\boldsymbol{j}_{1}, \boldsymbol{j}_{2}} \sigma_{z \boldsymbol{j}_{1}} \sigma_{z \boldsymbol{j}_{2}}
$$

and

$$
\Delta J=\left(J_{z}-J\right) / J
$$

is the measure of the surface induced anisotropy assumed to be the same for all lattice sites.

The Hamiltonian $\widehat{H}^{\prime}$ does not commute with $\widehat{H}_{0}$ since the latter can change the number of adjacent lattice points occupied by minus-spins. However, in the spirit of noninteracting spin-wave approximation we can calculate the eigenvalue of $\widehat{H}^{\prime}$ as if no two minus-spins ever occupied adjacent lattice sites

$$
\hat{H}^{\prime}|m, u\rangle=\frac{1}{2} \Delta J\left(N f_{t}-2 f_{t} m\right)|m, u\rangle,
$$

where $2 f_{t}$ is the number of nearest-neighbours in the thin film.

In this approximation $\hat{H}^{\prime}$ and $\widehat{H}_{0}$ commute, the eigenvalue of $\widehat{H}^{\prime}$ can be combined with the eigenvalue of $\epsilon_{H}$ and the partition function can be written in the form

$$
Z=\sum_{m=0}^{N} \exp \left[\beta\left(h_{m}^{\prime}+A_{m}\right)\right] \sum_{l=0}^{\infty} \frac{\beta^{l}}{l !} \sum_{u_{0}} s_{l}^{0}\left(m, u_{0}\right),
$$

where

$$
h_{m}^{\prime}=h_{m}+\frac{1}{2} \Delta J\left(N f_{t}-2 f_{t} m\right)
$$

We will consider the mono- and double layer as the assumption that the surface induced anisotropy is the same for all atoms is justified in these two cases. 


\subsection{Anisotropic monolayer}

The calculation of $s_{l}^{0}$ in the case of monolayer can be performed exactly in the same way as for a bulk ferromagnet [10]. We can write

$$
\begin{aligned}
& \sum_{u} s_{l}^{0}(m, u) \\
& \quad=\left(2 f_{m} m\right)^{i} \sum_{l_{1} \ldots l_{m}} L \sum_{p_{1} \ldots p_{\boldsymbol{q}}} P^{-1} \sum_{j_{1} \ldots \boldsymbol{j}_{m}} \prod_{k_{1}=1}^{p_{1}} W^{\left(k_{1}\right)}(1) \prod_{k_{2}=1}^{p_{2}} W^{\left(k_{2}\right)}(2) \ldots
\end{aligned}
$$

where

$$
\begin{aligned}
& L=\frac{1}{m^{l}} \frac{l !}{l_{1} ! l_{2} ! \ldots l_{m} !}, \\
& P=p_{1} ! p_{2} ! \ldots p_{q} ! 1^{p_{1}} 2^{p_{2}} \ldots q^{p_{q}}, \\
& l_{1}+l_{2}+\ldots+l_{m}=l, \\
& p_{1}+2 p_{2}+\ldots+q p_{q}=m, \\
& W^{(i)}(q)=W_{l_{i_{1}} l_{i_{2}} \ldots l_{i_{q}}}\left(j_{i_{1}}, j_{i_{2}}, \ldots j_{i_{q}}\right),
\end{aligned}
$$

$2 f_{m}$ is the number of the nearest-neighbours in the monolayer and $W^{(i)}(q)$ is the joint probability that the minus-spin initially at $j_{i_{1}}$ arrives after $l_{i_{1}}$ steps at $j_{i_{1}}$, the minus-spin initially at $j_{i_{2}}$ arrives after $l_{i_{2}}$ steps at $j_{i_{3}}$, etc., and finally the minus-spin initially at $j_{i_{q}}$ arrives after $l_{i_{q}}$ steps at $j_{i_{1}}$ restoring the original configuration [10]. The presence of the factor $P^{-1}$ accounts for the possibility of obtaining indistinguishable configurations by interchanging identical objects.

Similarly as for the bulk ferromagnet,

$$
\sum_{\boldsymbol{j}_{1} \ldots \boldsymbol{j}_{q}} W^{(i)}(q)=\sum_{\boldsymbol{j}_{1}} W_{l_{i_{1}}+\ldots l_{i_{q}}}\left(j_{1}\right)=N W_{l_{i_{1}}+\ldots l_{i_{q}}}\left(j_{1}\right),
$$

where $N$ is the number of lattice sites in the monolayer, and

$$
Z=\exp [\beta(a+\Delta J) N] \prod_{q} \exp \left[N q^{-1} \exp \left(-2 \beta a q-\beta \Delta J f_{m} q\right) G(\beta q)\right],
$$

where

$$
G(\beta q)=\Omega^{-1} \int \exp \left[-2 f_{m} \beta q\left(1-R_{\rho}\right)\right] \mathrm{d} \rho .
$$

The expression (3.14) differs from the corresponding formula for bulk isotropic ferromagnet in two points. Equation (3.14) contains $\Delta J$ which is the measure of the anisotropy and $R_{\rho}$ is to be calculated for two-dimensional lattice. It is easy to verify that for the sc lattice

$$
G(\beta q)=I_{0}^{2}(\beta q) \exp (-2 \beta q)
$$

where $I_{0}$ is the Bessel function.

Now we can write the expression for the free energy $\mathcal{F}=-k T \ln Z$ of the system and calculate the spontaneous magnetization using the standard relation

$$
M=-\frac{\mu}{N J}\left(\frac{\partial \mathcal{F}}{\partial a}\right)_{a \rightarrow 0} .
$$


Since the asymptotic expansion of the Bessel function for large argument is known, the low temperature expansion generalized for an arbitrary spin can be finally written in the following form:

$$
M / \mu=1-\frac{1}{2 \pi S} \frac{\zeta\left(\beta f_{m} \Delta J 2 S, 1\right) k}{4 S J} T-\ldots
$$

where

$$
\zeta(x, n)=\sum_{q=1}^{\infty} \frac{\exp (-x n q)}{q^{n}}
$$

replaces the Riemann $\zeta$ function appearing in the corresponding expression for an isotropic ferromagnet.

The function $\zeta(x, 1)$ does not diverge for $x>0(\Delta J>0)$ and reduces then to the elementary function

$$
\zeta(x, 1)=-\ln \left(1-\mathrm{e}^{-x}\right) .
$$

The effect of anisotropy on the spontaneous magnetization calculated from the expansion (3.18) truncated after first two terms is shown in Fig. 1. The figure shows that even a very small anisotropy can restore the spontaneous magnetization. The figure also shows that the spontaneous magnetization exhibits a quasi-linear behaviour in a certain temperature regions depending on the magnitude of the anisotropy.

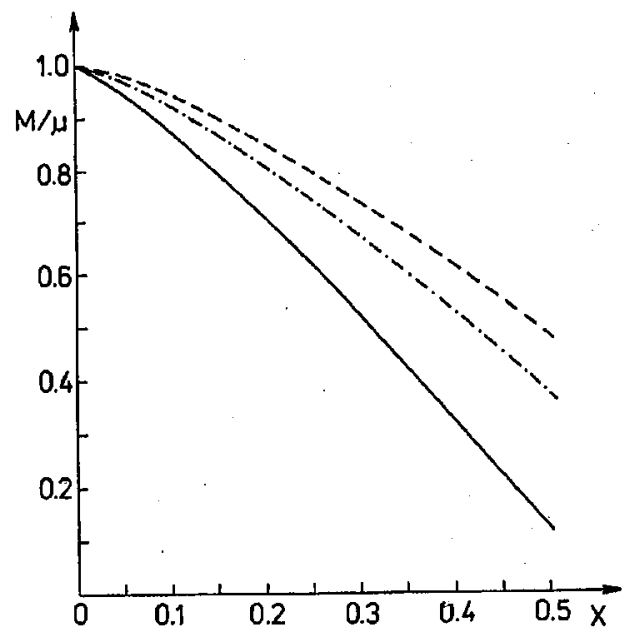

Fig. 1. The spontaneous magnetization as a function of temperature. $M$ and $\mu$ are the spontaneous magnetization per atom and the magnetic moment of each atom, respectively, $x=k T /\left[2(2 S)^{2} J\right]$ is the measure of the temperature. The solid line is obtained for $\Delta J=0.002$, the dashed-dotted line - for $\Delta J=0.01$ and the dashed line - for $\triangle J=0.02$. 


\subsection{Anisotropic double layer}

The calculation of $s_{l}^{0}$ can be performed using Eq. (3.7) provided that $f_{m}$ is replaced by the bulk value $f$ and $W^{k}(q)$ is expressed in terms of probabilities $T_{l}\left(j_{1}, j_{2}\right)$ that the minus-spin initially at $j_{1}$ arrives after $l$ steps at $j_{2}$ not crossing any of two surfaces of the double-layer

$$
W^{i}(q)=T_{l_{i_{1}}}\left(j_{1}, j_{2}\right) T_{i_{i_{1}}}\left(j_{2}, j_{3}\right) \ldots T_{i_{q}}\left(j_{q}, j_{1}\right) \text {. }
$$

Let us assume that we have the double-layer of the sc symmetry and (001) surface planes. It is convenient to decompose the random walk of a minus-spin into the unrestricted random walk within the plane parallel to the surface and the random walk in the perpendicular direction

$$
T_{7}\left(j_{1} j_{2}\right)=\sum_{k=0}^{l}\left(\begin{array}{l}
l \\
k
\end{array}\right)\left(\frac{f_{m}}{f}\right)^{l-k} P_{l-k}\left(j_{1 \|}, j_{2 \|}\right)\left(\frac{f_{p}}{f}\right)^{k} Q_{k}\left(n_{z_{1}}, n_{z_{2}}\right),
$$

where $f_{m}$ has the same meaning as in Eq. (3.7), $f_{m}+f_{p}=f, P$ and $Q$ are the probabilities for the random walk parallel and perpendicular to the surface, respectively, and $j=\left(j_{\|}, n_{z}\right)$.

The probability $Q_{n}$ for the random walk with absorbing traps was discussed in details in Refs. [16] and [17]. The problem of the random walk in the double layer can be reduced thus to two-dimensional unrestricted walk in the plane and one-dimensional walk with absorbing traps placed, say, at $n_{t_{1}}=-1$ and $n_{t_{2}}=2$. The generating function for the one-dimensional walk with the absorbing barriers can be written as

$$
\begin{aligned}
& Q\left(n_{z}, z\right)=G\left(n_{z}, z\right)+\left[(z-1) G\left(n_{z}-n_{t_{1}}, z\right)+\delta_{n_{z}, n_{t_{1}}}\right] Q\left(n_{t_{1}}, z\right) \\
& +\left[(z-1) G\left(n_{z}-n_{t_{2}}, z\right)+\delta_{n_{z}, n_{t_{2}}}\right] Q\left(n_{t_{2}}, z\right),
\end{aligned}
$$

where

$$
G(n, z)=x^{|n|}\left(1-z^{2}\right)^{-1 / 2}
$$

with

$$
x=\left[1-\left(1-z^{2}\right)^{1 / 2}\right] / z .
$$

Setting $n_{z}$ to $n_{t_{1}}$ and $n_{t_{2}}$ and solving resulting set of simultaneous equations we get after some rearrangements,

$$
\begin{aligned}
& Q(0, z)=\frac{4}{4-z^{2}}, \\
& Q(1, z)=\frac{1}{2} \frac{z}{1-(z / 2)^{2}},
\end{aligned}
$$

and

$$
Q_{k}\left(0, n_{z}\right)=\left[\frac{1}{k !} \frac{\mathrm{d}^{k}}{\mathrm{~d} z^{k}} Q\left(n_{z}, z\right)\right]_{z=0} .
$$

It can be easily shown that Eq. (3.13) is also fulfilled in the case of the double layer. The summation over $l_{i}$ involved in $W_{l_{i_{1}}+\ldots l_{i_{q}}}$ can be performed. The summations over $l_{i}$ and $l$ and over $p_{i}$ and $m$ subjected to the conditions (3.10) 
and (3.11), respectively, can be replaced by the independent summations from 0 to $\infty$ over $l_{i}$ and $p_{i}$ and the partition function can be written in the following form:

$$
Z=\exp [\beta(a+\Delta J) N] \prod_{q} \exp \left[N q^{-1} \exp \left(-2 \beta a q-\beta \Delta J f_{t} q\right) W(q),\right.
$$

where

$$
W(q)=N \exp \left(-2 f_{t} \beta q\right) \sum_{l=0}^{\infty} \frac{(2 f)^{l} q^{l} \beta^{l}}{l !} T_{l}\left(j_{0}, j_{0}\right) .
$$

Using Eq. (3.22) and taking into account that $P_{l}$ is the probability of return for the random walk on two-dimensional lattice,

$$
W(q)=N \exp \left(-2 f_{t} \beta q\right) I_{0}^{2}(2 \beta q) \sum_{k=0}^{\infty} \frac{\left(2 f_{p}\right)^{k} q^{k} \beta^{k}}{k !} Q_{k}(0,0) .
$$

Now, we can use again the representation (2.31) of the reciprocal of $k$ ! and write

$$
W(q)=N \exp \left(-2 f_{t} \beta q\right) I_{0}^{2}(2 \beta q) \frac{1}{2 \pi} \mathrm{e}^{\eta} \int_{-\infty}^{\infty} \mathrm{d} x \mathrm{e}^{-\mathrm{i} x} \frac{u}{\alpha_{p}} Q(0, u),
$$

where

$$
\begin{gathered}
u=\frac{\alpha_{p}}{\eta-\mathrm{i} x}, \\
\alpha_{p}=2 f_{i} q \beta .
\end{gathered}
$$

The integral in Eq. (3.32) is easy to calculate and

$$
W(q)=N \exp \left(-2 f_{t} \beta q\right) I_{0}^{2}(2 \beta q) \frac{1}{2}\left(\mathrm{e}^{\beta q}+\mathrm{e}^{-\beta q}\right) .
$$

The asymptotic expansion of $W(q)$ can be now obtained and the spontaneous magnetization, generalized for an arbitrary spin, can be finally written in the form

$$
M / \mu=1-\frac{1}{2 \pi S} \frac{1}{2} \frac{\zeta\left(\beta f_{t} \Delta J 2 S, 1\right) k}{4 S J} T-\ldots
$$

The above equation differs from the expansion (3.18) for the monolayer by the presence of the factor one half in the second term. The argument of the $\zeta$ function is also slightly different. If we, however, re-scale the coordinate in Fig. 1 and $\Delta J$ we can use this figure to illustrate the behaviour of the spontaneous magnetization as a function of temperature also for the double layer.

\section{Conclusions}

The random walk technique was used to derive the full low temperature expansion of the spontaneous magnetization for the semi-infinite Heisenberg ferromagnet. The derivation is exact within the scope of the noninteracting spin-wave approximation. The spontaneous magnetization in the surface layer for semi-infinite ferromagnet starts with the term $T^{3 / 2}$ in agreement with previous results. It was also shown that the spontaneous magnetization of anisotropic ultrathin film ferromagnets exhibits quasi-linear behaviour in certain temperature regions depending on the magnitude of the exchange anisotropy. 


\section{Appendix}

\section{Derivation of the expression for $\left\langle S_{z, 1}\right\rangle$}

Equation (4.2) of Ref. [12] assumes the following form:

$$
\begin{aligned}
Z= & \exp \left[\beta(a+f) N-K f_{\mathrm{d}} / 2\right] \\
& \times \prod_{q}\left(\sum_{p_{q}=0}^{\infty} \frac{1}{p_{q} !}\left[q^{-1} \exp (-2 \beta q(a+f)) \sum_{t=0}^{\infty} T^{(t)}(q)\right]^{p_{q}}\right),
\end{aligned}
$$

where now the joint probability $T^{(t)}(q)$ that $q$ minus-spins forming $q$-ring restore their initial configuration passing $t$ times by the surface plane is given by

$$
T^{(t)}(q)=\sum_{\boldsymbol{j}_{0}} T^{(t)}\left(q, j_{0}\right)
$$

where

$$
T^{(t)}(j, q)=\sum_{l=0}^{\infty} \frac{(2 f)^{l} q^{l} \beta^{l}}{(l+t) !} \sum_{l_{1}+\ldots l_{t+1}=l} \sum_{j_{s_{1}}, \ldots j_{s_{t}}} T_{l_{1}}\left(j_{0}, j_{s_{1}}\right) \ldots T_{l_{t+1}}\left(j_{s_{t}}, j_{0}\right)
$$

and $j_{s_{i}}$ denote the lattice sites belonging to the surface layer. The operator $\sigma_{z j}$ appearing in Eq. (2.5), according to Eqs. (2.16)-(2.12), fixes successively the positions of minus-spins in the configuration $u_{0}$. Collecting together the terms which correspond to $q_{i}$ ring we obtain $p_{q_{i}} q_{i}$ identical terms and the local mean spin value can be written as

$$
\begin{aligned}
& \left\langle S_{z \boldsymbol{j}}\right\rangle=\frac{1}{2}-\lim _{a \rightarrow 0} Z^{-1} \\
& \times \sum_{q_{i}} \prod_{q \neq q_{i}}\left[\sum_{p_{q}=0}^{\infty} \frac{1}{p_{q} !}\left(q^{-1} \exp [-2 \beta q(a+f)] \sum_{t=0}^{\infty} T^{(t)}(q)\right)^{p_{q}}\right] \\
& \times\left[\sum_{p_{q_{i}}=1}^{\infty} \frac{1}{\left(p_{q}-1\right) !}\left(q^{-1} \exp \left[-2 \beta q_{i}(a+f)\right] \sum_{t=0}^{\infty} T^{(t)}\left(q_{i}\right)\right)^{p_{q_{i}}-1}\right. \\
& \left.\quad \times \exp \left[-2 \beta q_{i}(a+f)\right] \sum_{t=0}^{\infty} T^{(t)}\left(j, q_{i}\right)\right] .
\end{aligned}
$$
Performing the summation over $p_{q}$ and generalizing the result for the case of
$S>1 / 2$ we get

where

$$
\left\langle S_{z \boldsymbol{j}}\right\rangle=\dot{S}-\sum_{q=1}^{\infty} \exp (-4 f S \beta q) \sum_{t=0}^{\infty} T_{S}^{(t)}(j, q)
$$

$$
\begin{aligned}
& T_{S}^{(t)}(j, q) \\
& \quad=\sum_{l=0}^{\infty} \frac{(2 f)^{l}(2 S q)^{l} \beta^{l}}{(l+t) !} \sum_{l_{1}+\ldots l_{t+1}=l} \sum_{j_{s_{1}}, \ldots j_{s_{t}}} T_{l_{1}}\left(j_{0}, j_{s_{1}}\right) \ldots T_{l_{t+1}}\left(j_{s_{t}}, j_{0}\right) .
\end{aligned}
$$


Using Eqs. (2.20) and (2.21) and performing summations over $j_{s_{i}}$ we get the result which depends only on the position of crystal layer with respect to the surface layer and can be written ir the form of Eq. (2.24).

The use of the repiescitation (2.31) of the reciprocal of $n$ ! allows us to perform the summations over $i_{k}$ and $t$ in Eqs. (2.25) and (2.23) and to reduce (2.24) to the expression

$$
\begin{aligned}
\left\langle S_{\boldsymbol{z} \boldsymbol{j}}\right\rangle & =S-\sum_{q=1}^{\infty} \exp (-2 f \alpha) I_{0}(2 \alpha)^{2} \exp (2 \alpha) \\
& \times \int_{-\infty+\mathrm{i} \eta /(2 \alpha)-\mathrm{i}}^{\infty+\mathrm{i} \eta /(2 \alpha)-\mathrm{i}}(-2 \pi \mathrm{i} \alpha x)\left(\frac{\sqrt{x+2 \mathrm{i}}}{\sqrt{x}}-1\right) .
\end{aligned}
$$

The Bessel functions result from the integration over $\mathrm{d} \rho_{x} \mathrm{~d} \rho_{y}$ and it can be easily shown by contour integration that the above equation can be reduced to Eq. (2.33).

\section{References}

[1] See for example, E. Bruno, B.L. Gyorffy, Appl. Surf. Sci. 75, 320 (1994) and references therein.

[2] See for example, H. Tang, Z.Q. Qiu, Y.W. Du, G.P. Stern, J.C. Walker, J. Appl. Phys. 63, 3659 (1988).

[3] D.L. Mills, A.A. Maradudin, J. Phys. Chem. Solids 28, 1855 (1967).

[4] D.L. Mills, Phys. Rev. B 40, 11153 (1989).

[5] J. Mathon, NATO ASI Ser. B 345, 113 (1995).

[6] H.J. Elmers, J. Hauschild, U. Gradmann, Phys. Rev. B 54, 15224 (1996).

[7] Ar. Abanov, V. Kalatsky, V.L. Pokrovsky, Phys. Rev. B 51, 1023 (1995).

[8] N.D. Mermin, H. Wagner, Phys. Rev. Lett. 17, 1133 (1966).

[9] M. Bander, D.L. Mills, Phys. Rev. B 38, 12015 (1988).

[10] W. Opechowski, Physica 25, 476 (1959).

[11] H.A. Kramers, Commun. Kamerlingh Onnes Lab., Suppl. No. 83, Leiden 1936.

[12] P. Modrak, Physica 72, 43 (1974).

[13] S. Chandrasekhar, Rev. Mod. Phys. 15, 1 (1943).

[14] F. Cyrot-Lackmann, Surf. Sci. 15, 535 (1969).

[15] M.J. Lighthill, Introduction to Fcurier Analysis and Generalized Functions, Cambridge 1959.

[16] M.N. Barber, B.W. Ninham, Random and Restricted Walks, Gordon and Breach, New York 1970.

[17] E.W. Montroll, G.H. Weiss, J. Math. Phys. 6, 167 (1965). 